\title{
The influence of axle position and the use of accessories on the activity of upper limb muscles during manual wheelchair propulsion
}

\author{
Guilherme da Silva Bertolaccini, Idinei Francisco Pires de Carvalho Filho, \\ Gustavo Christofoletti, Luis Carlos Paschoarelli \& Fausto Orsi Medola
}

To cite this article: Guilherme da Silva Bertolaccini, Idinei Francisco Pires de Carvalho Filho, Gustavo Christofoletti, Luis Carlos Paschoarelli \& Fausto Orsi Medola (2018) The influence of axle position and the use of accessories on the activity of upper limb muscles during manual wheelchair propulsion, International Journal of Occupational Safety and Ergonomics, 24:2, 311-315, DOI:

10.1080/10803548.2017.1294369

To link to this article: https://doi.org/10.1080/10803548.2017.1294369

Accepted author version posted online: 17

Feb 2017.

Published online: 24 Mar 2017.

Submit your article to this journal $\widetilde{ }$

Lll Article views: 170

View Crossmark data ¿

Citing articles: 3 View citing articles $[\pi$ 


\title{
The influence of axle position and the use of accessories on the activity of upper limb muscles during manual wheelchair propulsion
}

\author{
Guilherme da Silva Bertolaccinia ${ }^{\mathrm{a}}$, Idinei Francisco Pires de Carvalho Filho ${ }^{\mathrm{b}}$, Gustavo Christofolettic \\ Luis Carlos Paschoarelli ${ }^{\mathrm{a}, \mathrm{b}}$ and Fausto Orsi Medola (iD) ${ }^{\mathrm{a}, \mathrm{b} *}$ \\ ${ }^{a}$ Graduate Programme in Design, UNESP - University Estadual Paulista, Brazil; ${ }^{b}$ Department of Design, UNESP - University \\ Estadual Paulista, Brazil; ${ }^{c}$ Department of Physiotherapy, UFMS - Federal University of Mato Grosso do Sul, Brazil
}

\begin{abstract}
Introduction. Wheelchair configuration is an important factor influencing the ergonomics of the user-device interface and, from a biomechanical point of view, small changes in chair setup may have a positive influence on the demand on the upper limbs during manual propulsion. This study aimed to investigate the influence of the position of the rear wheels' axle and the use of accessories on the activity of upper limb muscles during manual wheelchair propulsion. Methods. Electromyography signals of the biceps, triceps, anterior deltoids and pectoralis major were collected for 11 able-bodied subjects in a wheelchair propulsion protocol with four different wheelchair configurations (differing in axle position and the use of accessories) on a straightforward sprint and a slalom course. Results. With accessories, moving the axle forward led to a decrease in the activity of all muscles in both the straightforward sprint (significant differences in triceps, anterior deltoids and biceps) and the slalom course (significant difference in anterior deltoids and biceps). However, when propelling the chair without accessories, no difference was found related to axle position. Conclusion. Changes in wheelchair configuration can influence the ergonomics of manual wheelchair propulsion. Reducing the biomechanical loads may benefit users' mobility, independence and social participation.
\end{abstract}

Keywords: wheelchairs; biomechanics; manual propulsion; ergonomics; assistive technologies

\section{Introduction}

Wheelchair use has been addressed in a variety of topics covering the biomechanics of manual propulsion [1-3], sports $[4,5]$, problems related to mobility $[6,7]$ and workplace [8-10]. Although used widely, the wheelchair has problems that prevent the user from moving with independence, safety and satisfaction. From an ergonomic point of view, such problems can be divided into two main topics: seating and mobility. While the former focuses on the interaction between the user's body and the support interfaces (seat, backrest, armrest and foot support), mobility addresses the user's actions and the resulting movement of the wheelchair.

Manual wheelchairs users have many functional difficulties in daily life. Mobility tasks as simple as climbing a sidewalk can be very hard. In this way, moving with the wheelchair for long distances is strongly limited and, as a result, the daily distance traveled and average speed are significantly lower in wheelchair mobility when compared with walking [11-14]. Manual wheelchair propulsion exposes the upper limbs to a harmful combination of load and repetition that may cause many injuries [15]. Because users occupy the chair for about $11 \mathrm{~h} /$ day [14], the wheelchair becomes an extension of the users' body and influences their social participation [16] and daily work.

In order to study wheelchair mobility in a closer way to how people move in their daily routine, it is important to investigate the biomechanics of manual propulsion not only in straightforward motion, but also in trajectories comprising changes in movement direction and acceleration. A previous study demonstrated that the influence of the mechanical aspects on the movement of the wheelchair is dependent on the trajectory and acceleration [17].

The wheelchair configuration is very important to determine the upper limb actions during manual propulsion. The position and camber of the rear wheels, tire and wheel types, seat and backrest angles, frame design and the use of accessories (armrest, clothing shields) are features of the equipment design that can affect propulsion forces, upper limb range of motion, rolling resistance and system stability [18]. Therefore, a proper selection regarding the wheelchair configuration can improve overall mobility performance.

Many wheelchairs have adjustable settings allowing the users to set up the wheelchair in accordance with their needs and preferences. Moving the rear wheels forward or rearward and using or removing accessories such as

\footnotetext{
*Corresponding author. Email: fausto.medola@faac.unesp.br
} 
armrests and seat cover plates are two changes in configuration commonly made by users to make the equipment more suitable to them. However, the influence of the combination between axle position and use of accessories on the biomechanics of manual propulsion is still unclear. This information may contribute to improve the ergonomics of manual wheelchairs by providing objective data on how changes in the equipment configuration influence the users' actions during manual wheelchair propulsion.

This study aimed at investigating the influence of the position of the rear wheels' axle and the use of accessories on the activity of upper limb muscles during manual wheelchair propulsion.

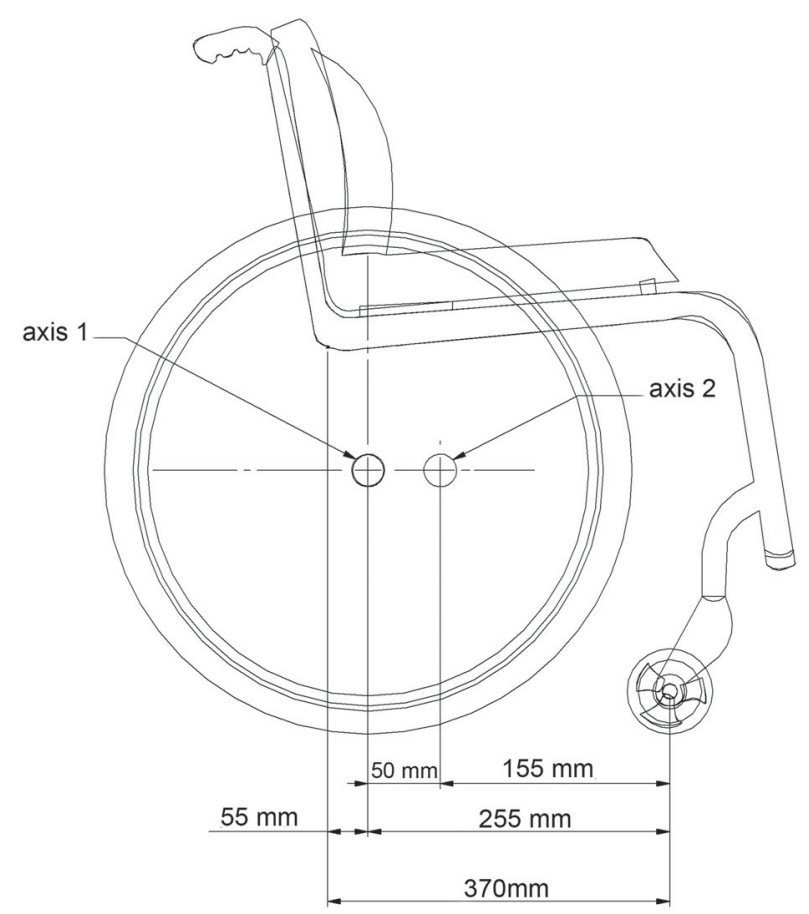

Figure 1. Relative position of the rear wheels to the user.

\section{Materials and methods}

\subsection{Participants}

A convenience sample of 11 participants without physical disabilities (mean age $23.82 \pm 3.46$ years, mean height $1.77 \pm 0.06 \mathrm{~m}$, mean weight $76.09 \pm 11.43 \mathrm{~kg}$ ), all male, were recruited at the São Paulo State University (UNESP, Bauru, Brazil) and voluntarily participated in this study. Participants met the following inclusion criteria: (a) minimum age of 18 years; (b) had no upper limb pain, injuries or disorders that could influence the manual wheelchair propulsion. Prior to data collection, participants read and signed an informed consent form that had been submitted and approved by the Ethics Committee of the Faculty of Architecture, Arts and Communication - UNESP (Process. N. 800.500).

\subsection{Equipment and procedure}

A manual wheelchair with a rigid frame was used in four different configurations, varying according to a relative fore-aft rear axle position to the frame of $50 \mathrm{~mm}$ (Figure 1) and the use or not of accessories (clothing shields and armrests), as shown in Figure 2. The total weight of the accessories was $1.95 \mathrm{~kg}$.

These configurations were tested in two different trajectories: straightforward sprint (acceleration in a $15-\mathrm{m}$ straight motion) and slalom course (nine cones aligned and separated by decreasing distances) (Figure 3), as proposed in a previous study [17]. For both trajectories, subjects were instructed to propel the chair as fast as possible. The sequence of trajectories and wheelchair configurations were randomized for each subject.

During the tests, surface electromyography (EMG) data of the biceps brachial, triceps brachial, anterior deltoids and pectoralis major were collected using four-channel wireless sensors (T-Sens; TEA Ergo, France) and a Datalogger module (CAPTIV; TEA Ergo, France) to register the EMG signals. Triode surface self-adhesive electrodes T3402M (Thought Technology, Canada) were placed in the

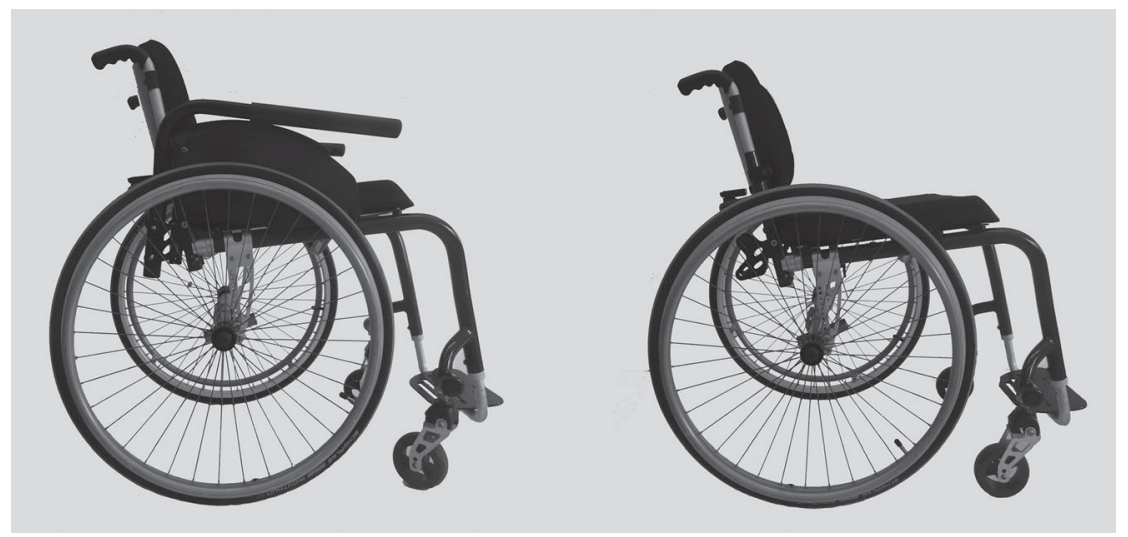

Figure 2. Wheelchairs with and without accessories. 


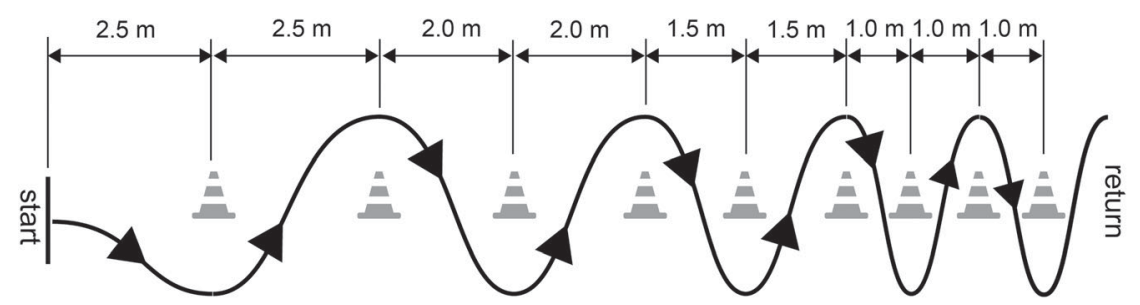

Figure 3. Slalom course.

Table 1. Mean EMG activity $(\mathrm{mV})$ of the four muscles during manual wheelchair propulsion with the accessories.

\begin{tabular}{lcccc}
\hline Muscle & Trajectory & Rearward axle & Forward axle & $p$ \\
\hline Pectoralis major & Straightforward & $101.64(84.55)$ & $89.36(63.82)$ & 0.24 \\
& slalom & $35.71(24.10)$ & $34.19(28.09)$ & 0.68 \\
Triceps brachial & Straightforward & $190.46(161.61)$ & $145.79(99.81)$ & $0.02^{*}$ \\
& slalom & $91.44(58.42)$ & $87.45(45.18)$ & 0.57 \\
Anterior deltoids & Straightforward & $281.16(159.22)$ & $224.37(110.98)$ & $0.04^{*}$ \\
& slalom & $124.23(56.12)$ & $98.29(52.11)$ & $0.01^{*}$ \\
Biceps brachial & Straightforward & $152.84(56.42)$ & $96.57(48.31)$ & $0.02^{*}$ \\
& slalom & $53.13(37.88)$ & $36.32(18.59)$ & $0.01^{*}$ \\
\hline
\end{tabular}

$* p<0.05$.

Note: $\mathrm{EMG}=$ electromyography.

respective positions for each muscle in accordance with the SENIAM project (www.seniam.org) on the dominant side of the subject's body to record the electrical activity of the muscles. Surface EMG data were sampled at 2048 $\mathrm{Hz}$ (which is more than double the frequency in human muscles) [19] to satisfy the Nyquist Theorem, and the root mean square (rms) value was calculated and transmitted by the T-Sens module. The frequency of the rms calculation was $128 \mathrm{~Hz}$, and data analysis was performed with the CAPTIV L-7000 software (TEA Ergo, France). All EMG calculation was in $\mathrm{mV}$ and analyzed during the complete trajectory, and the first and last pushes were discarded, in order to analyze the muscle activity in plain motion. The mean time window of the analysis was $6.52 \mathrm{~s}$ for the straightforward motion and $31.23 \mathrm{~s}$ for the slalom.

\subsection{Data analysis}

Mean values were obtained for the EMG measurements of the four muscles of all subjects. To verify statistical differences in the mean EMG of the four muscles between all of the wheelchair configurations for both trajectories, Friedman's test was applied. To verify statistical difference in paired data, the Wilcoxon test was applied, because the data did not show a normal distribution as revealed by the Shapiro-Wilk test. Significance was determined by $p \leq 0.05$. All statistical analyses were performed using SPSS version 22.0.

\section{Results}

The results show that wheelchair design and configuration can influence the load on the upper limbs. For the wheelchair with accessories, axle position was shown to be a factor that influences the biomechanical load on the upper limbs. In the straightforward sprint, the axle in the forward position showed lower activity than the rearward position in all of the muscles, with significant difference found in the triceps $(p=0.02)$, anterior deltoids $(p=0.04)$ and biceps $(p=0.02)$. This was also found in the slalom course, because propelling the chair with the axle in the forward position required less activity of all muscles in comparison with the rearward position, although significant difference was found only with the anterior deltoids $(p=0.01)$ and biceps $(p=0.01)$ ( Table 1$)$.

Conversely, the influence of axle position appeared to be minimized when propelling the chair with the accessories removed, because there was no significant difference regarding the axle position for both the straightforward sprint and the slalom course (Table 2).

The differences in the muscle activity related to the wheelchair configurations (axle position and accessories) and trajectories can be interpreted by the ratio of the mean EMG for the forward and rearward axle positions, as presented in Figure 4. Values lower than one indicate lower demand on the upper limbs during manual propulsion with the axle in a forward position, while values higher than one indicate the opposite, i.e., lower demand with the axle in a rearward position.

\section{Discussion}

Wheelchair design and configuration are important factors that can influence the demand on the upper limbs during manual propulsion. This study addressed the influence of a 
Table 2. Mean EMG activity (mV) of the four muscles during manual wheelchair propulsion without the accessories.

\begin{tabular}{lcccc}
\hline Muscle & Trajectory & Rearward axle & Forward axle & $p$ \\
\hline Pectoralis major & Straightforward & $88.66(71.58)$ & $90.14(82.33)$ & 0.99 \\
& slalom & $31.56(24.85)$ & $29.39(19.63)$ & 0.49 \\
Triceps brachial & Straightforward & $183.35(150.79)$ & $192.24(1342.43)$ & 0.37 \\
& slalom & $91.12(56.36)$ & $95.07(54.59)$ & 0.33 \\
Anterior deltoids & Straightforward & $253.41(131.53)$ & $255.01(150.73)$ & 0.92 \\
& slalom & $105.45(54.30)$ & $99.93(49.05)$ & 0.43 \\
Biceps brachial & Straightforward & $109.78(70.51)$ & $123.74(95.91)$ & 0.30 \\
& slalom & $40.93(25.82)$ & $39.64(19.73)$ & 0.99 \\
\hline
\end{tabular}

Note: $\mathrm{EMG}=$ electromyography.

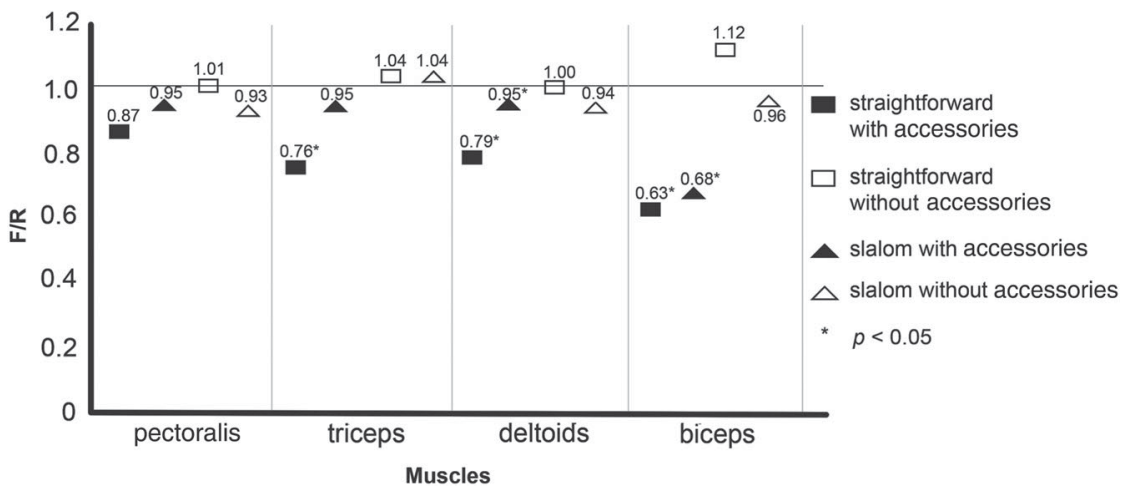

Figure 4. Ratio (F/R) of the mean EMG for the forward (F) and rearward (R) axle positions.

Note: $\mathrm{EMG}=$ electromyography.

slight but relevant aspect - axle position - on the activity of upper limb muscles that many times is neglected during equipment design and setup. Because some users remove the accessories (armrest and clothing shields) in order to make the chair lighter, we investigated the influence of axle position in both situations: with and without accessories.

Our results show that moving the axle position forward led to a decrease in the activity of upper limbs during manual wheelchair propulsion in both the straightforward sprint and the slalom course. However, this difference was significant only in the standard configuration (with accessories). Previous studies have highlighted the benefits of positioning the axle forward on the demand on the upper limbs [20,21]. From a mechanical perspective, moving the axle forward reduces the length of the equipment, which impacts the rotational inertia of the system. Additionally, moving the rear wheels forward may contribute to wheelchair mobility by reducing the rolling resistance, because the user's body moves toward the rear wheels [22].

Although moving the rear wheels' axle forward seems to provide an ergonomic benefit for the user in terms of facilitating manual propulsion, it must be taken into account that it has consequences on the equipment stability. In practice, the more forward the axle, the easier it is for the wheelchair to tip over. The guidelines for preserving upper limb function after spinal cord injury recommend that the rear wheels' axle must be positioned as forward as possible without compromising equipment stability [19].
This can only be set up with the participation of the user.

Although the current study produced important findings, it has limitations that must be noted. First, procedures were not performed with real users, and only men participated in the study. Additionally, tasks were conducted at the highest speed the subjects could propel. Another limitation is that we did not assess the subjects' perceptions of the efforts during manual wheelchair propulsion, which could demonstrate whether these changes in wheelchair design and configuration are perceived or not by the subjects.

\section{Conclusion}

The wheelchair can enhance users' mobility, independence and social participation, and small changes in its design and configuration can lead to important contributions to daily mobility. The current findings suggest that moving the rear wheels' axle forward may have a positive effect on manual propulsion by decreasing the activity of upper limb muscles, although this was only found with the chair in its standard configuration (with accessories). This shows an existing correlation between wheelchair mechanics and propulsion biomechanics. From an ergonomic perspective, adjusting the equipment mechanics (resulting from different configurations) must be seen as a strategy to reduce the loads on the upper limbs, thus benefiting users' mobility. 
This information may benefit designers, manufacturers and health professionals in the design, prescription and provisioning of manual wheelchairs. Providing a wheelchair with adjustable configurations - the current study focused on the axle position - may help the user to set up the chair in accordance with his/her characteristics, needs and expectations.

\section{Disclosure statement}

No potential conflict of interest was reported by the authors.

\section{Funding}

This work was supported by CAPES (Coordination for the Improvement of Higher Level Personnel); FAPESP (São Paulo Research Foundation) [grant no. 16/05026-6]; CNPq (National Council for Scientific and Technological Development) [grant no. 458740/2013-6].

\section{Ethical approval}

All the study procedures were submitted and approved by the Ethics Committee of the Faculty of Architecture, Arts and Communication - UNESP (Process. N. 800.500).

\section{ORCID}

Fausto Orsi Medola (D) http://orcid.org/0000-0003-2308-6524

\section{References}

[1] Slowik JS, Requejo PS, Mulroy SJ, et al. The influence of wheelchair propulsion hand pattern on upper extremity muscle power and stress. J Biomech. 2016;49(9):1554-1561. doi:10.1016/j.jbiomech.2016.03.031

[2] Moon Y, Chandrasekaran J, Hsu IMK, et al. Variability of peak shoulder force during wheelchair propulsion in manual wheelchair users with and without shoulder pain. Clin Biomech (Bristol, Avon). 2013;28(0):1-16.

[3] Desroches G, Dumas R, Pradon D, et al. Upper limb joint dynamics during manual wheelchair propulsion. Clin Biomech (Bristol, Avon). 2010;25(4):299-306. doi:10.1016/j.clinbiomech.2009.12.011

[4] Aytar A, Zeybek A, Pekyavas NO, et al. Scapular resting position shoulder pain and function in disabled athletes. Prosthet Orthot Int. 2015;39(5):390-396. doi: 10.1177/0309364614534295

[5] Cavedon V, Zancanaro C, Milanese C. Kinematic analysis of the wheelchair tennis serve: implications for classification. Scand J Med Sci Sports. 2014;24(5):e381-e388. doi:10.1111/sms.12182

[6] Tomsone S, Haak M, Löfqvist M. Experiences of mobility device use over time: a multiple case study among very old Latvian women. Scand J Occup Ther. 2016;23(1):67-78. doi:10.3109/11038128.2015.1068850
[7] Brandt A, Kreiner S, Iwarsson S. Mobility-related participation and user satisfaction: construct validity in the context of powered wheelchair use. Disabil Rehabil Assist Thechnol. 2010;5(5):305-313. doi:10.3109/17483100903394636

[8] Das B, Black NL. Isometric pull and push strengths of paraplegics in the workspace: 1 . Strength measurement profiles. Int J Occup Saf Ergon. 2000;6(1):47-65. doi:10.1080/ 10803548.2000.11076443

[9] Das B, Black NL. Isometric pull and push strengths of paraplegics in the workspace: 2. Statistical analysis of spatial factors. Int J Occup Saf Ergon. 2000;6(1):67-80. doi:10.1080/10803548.2000.11076444

[10] Troy BS, Cooper RA, Robertson RN, et al. An analysis of work posture of manual wheelchair users in the office environment. J Rehabil Res Dev. 1997;34(2):151-161.

[11] Bohannon RW. Number of pedometer-assessed steps taken per day by adults: a descriptive meta-analysis. Phys Ther. 2007;87:1642-1650. doi:10.2522/ptj.20060037

[12] Karmarkar AM, Collins DM, Kelleher A, et al. Manual wheelchair-related mobility characteristics of older adults in nursing homes. Disabil Rehabil Assist Technol. 2010;5:428-437. doi:10.3109/17483107.2010.481346

[13] Tolerico ML, Ding D, Cooper RA, et al. Assessing mobility characteristics and activity levels of manual wheelchair users. J Rehabil Res Dev. 2007;44:561-571. doi:10.1682/JRRD.2006.02.0017

[14] Sonenblum SE, Sprigle S, Lopez RA. Manual wheelchair use: bouts of mobility in everyday life. Rehabil Res Pract. 2012. doi:10.1155/2012/753165

[15] Alm M, Saraste H, Norrbrink C. Shoulder pain in persons with thoracic spinal cord injury: prevalence and characteristics. J Rehabil Med. 2008;40:277-283. doi:10. 2340/16501977-0173

[16] Chaves ES, Boninger ML, Cooper R, et al. Assessing the influence of wheelchair technology on perception of participation in spinal cord injury. Arch Phys Med Rehabil. 2004;85:1854-1858. doi:10.1016/j.apmr.2004.03.033

[17] Medola FO, Dao PV, Caspall JJ, et al. Partitioning kinetic energy during freewheeling wheelchair maneuvers. IEEE Trans Neural Rehabil Eng. 2014;22(2):326-333. doi:10.1109/TNSRE.2013.2289378

[18] Medola FO, Elui VMC, Santana CS, et al. Aspects of manual wheelchair configuration affecting mobility: a review. J Phys Ther Sci. 2014;26(2):313-318. doi:10.1589/ jpts. 26.313

[19] Winter DA. Electromyogram recording, processing, and normalization: procedures and considerations. J Hum Muscle Perform. 1991;1(2):5-15.

[20] Paralyzed Veterans of America Consortium for Spinal Cord Medicine. Preservation of upper limb function following spinal cord injury: a clinical practice guideline for healthcare professionals. J Spinal Cord Med. 2005;28(5):434-470. doi:10.1080/10790268.2005.11753844

[21] Gorce P, Louis N. Wheelchair propulsion kinematics in beginners and expert users: influence of wheelchair settings. Clin Biomech (Bristol, Avon). 2012;27:7-15. doi:10.1016/j.clinbiomech.2011.07.011

[22] MacPhee AH, Kirby RL, Bell AC, et al. The effect of knee-flexion angle on wheelchair turning. Med Eng Phys. 2001;23:275-283. doi:10.1016/S1350-4533(01)00024-8 